\title{
PACLOBUTRAZOL NO FLORESCIMENTO E NA PRODUÇÃO DA MANGUEIRA 'TOMMY ATKINS'
}

\author{
Paclobutrazol on flowering and production of 'Tommy Atkins' mango
}

\author{
Nelson Fonseca ${ }^{2}$, Manoel Teixeira de Castro Neto $^{2}$, Carlos Alberto da Silva Ledo ${ }^{2}$
}

\section{RESUMO}

Técnicas eficientes de controle da época de produção são importantes para o sucesso econômico no cultivo de mangueira (Mangifera indica L.). Neste estudo, avaliaram-se o florescimento e a produção da mangueira 'Tommy Atkins' em resposta à aplicação de paclobutrazol (PBZ) no solo (2 mL do i.a./planta), e foliar em diferentes doses (0,5, 1, 1,5 e $2 \mathrm{~mL}$ do i.a. em aplicação única e em duas aplicações) sob condições de cultivo irrigado, na região semi-árida do Nordeste do Brasil. As variáveis estudadas foram percentual de florescimento, número de frutos e produção por planta, sendo avaliadas em três épocas após a aplicação do regulador de crescimento (68 a 110, 131 a 179 e 190 a 216 dias após para o florescimento da planta; e 162 a 203, 256 a 287 e 327 a 337 para o número e produção de frutos por planta). O PBZ aplicado no solo determinou percentuais de florescimento, número de frutos e produção por planta superiores à testemunha (água foliar) e aos tratamentos com PBZ foliar, além de antecipar o florescimento das plantas. O PBZ foliar, independente do número de aplicação e doses usadas, não superou significativamente a testemunha. O PBZ aplicado no solo antecipou o florescimento em 23 dias, em relação à testemunha e aos tratamentos com PBZ foliar, na segunda época de avaliação.

Termos para indexação: Mangifera indica, PBZ, regulador de crescimento.

\begin{abstract}
Efficient techniques for controlling the production time are important for the economical success in the mango cultivation. In this study, it was evaluated the flowering and production of mango fruit, variety Tommy Atkins, in response to the paclobutrazol application (PBZ), either in the soil ( $2 \mathrm{~mL}$ of the active ingredient per plant), or on foliage at different doses $(0.5,1,1.5$ and $2 \mathrm{~mL}$ of a.i. per plant in single or two applications), under conditions of irrigated cultivation, in the semi-arid area of Northeastern of Brazil. The studied variables were percentile flowering, number of fruits, and production per plant, being appraised in three times after the application of the growth regulator (68 to 110, 131 to 179 and 190 to 216 days after, for the flowering of the plant; and 162 to 203, 256 to 287 and 327 to 337 days after, for the number and production of fruits for plant). The application of PBZ in the soil determined percentile of flowering, number of fruits and production for plant superiors to the control (water to foliage) and to the treatments with PBZ to foliage, besides anticipating the flowering of the plants. PBZ to foliage, independent of the application number and used doses, didn't overcome the control significantly. Applied PBZ in the soil anticipated the flowering in 23 days, in relation to the control and to the treatments with PBZ to foliage, in the second evaluation time.
\end{abstract}

Index terms: Mangifera indica, PBZ, growth regulator.

(Recebido para publicação em 13 de janeiro de 2003 e aprovado em 26 de junho de 2003)

\section{INTRODUÇÃO}

O uso de substâncias reguladoras de crescimento vegetal adquiriu grande importância na agricultura e no cultivo da mangueira em especial, possibilitando modificar os processos que estimulam o florescimento e a subseqüente produção de frutos em períodos de baixa oferta do produto nos mercados interno e externo.

\footnotetext{
1. Parte da Tese de Doutorado do primeiro autor apresentada à Universidade Federal de Lavras/UFLA, Caixa Postal 37 - $37200-000$ - Lavras, MG, para a obtenção do título de Doutor em Fitotecnia.

2. Pesquisador da EMBRAPA Mandioca e Fruticultura. Rua Embrapa, s/n, Caixa Postal 07 - $44380-000$ - Cruz das Almas, BA. nelson@cnpmf.embrapa.br, castro@cnpmf.embrapa.br, ledo@cnpmf.embrapa.br.
} 
Atualmente, o uso de inibidores de crescimento, como o paclobutrazol (PBZ), tornou-se uma prática importante para a indução do florescimento e produção comercial de manga em todo território nacional, principalmente na Região Nordeste.

Em diversos estudos tem-se evidenciado que a aplicação do PBZ ao solo e subseqüente absorção pelas raízes resultam em efeitos mais acentuados sobre o florescimento e a produção da mangueira, do que a sua aplicação foliar (BURONDKAR et al., 1996; AGUIAR, 2001). No entanto, Oosthuyse e Jacobs (1996) observaram que a aplicação de PBZ via solo nas doses de 0,25 $\mathrm{mL}$ e $2,5 \mathrm{~mL} /$ planta não refletiu em aumentos no número de frutos e na produção das mangueiras 'Sensation' e 'Tommy Atkins' com dois anos de idade. Foi observada uma redução, tanto no número como na produção de frutos de plantas da variedade Tommy Atkins, em resposta à aplicação do produto na dose maior.

Com relação ao fracionamento das doses de PBZ aplicados via foliar, os resultados têm sido divergentes. Na Tailândia, Tongumpai et al. (1996) observaram os efeitos de aplicações foliares simples e múltiplas de PBZ a 1000 e $2000 \mathrm{mg} / \mathrm{L}$ sobre a porcentagem de florescimento e os comprimentos de panículas e ramos da variedade Nam Dok Mai. Eles observaram que duas aplicações do produto determinaram a antecipação e uniformização do florescimento e o aumento do número de panículas florais. Nas condições da Região Nordeste do Brasil, Reis et al. (2000) constataram que a porcentagem de florescimento, o número de frutos e a produção não foram afetados significativamente pela aplicação de PBZ via foliar, associado ou não ao ethephon.

Objetivou-se com este estudo determinar os efeitos do PBZ sobre o florescimento e a produção da mangueira 'Tommy Atkins' em resposta a diferentes formas, doses e números de aplicações do regulador de crescimento, nas condições do semi-árido do Nordeste do Brasil.

\section{MATERIAL E MÉTODOS}

O trabalho foi realizado numa propriedade do Núcleo-8 do Perímetro Irrigado Senador Nilo Coelho, Petrolina, PE, localizada a $9^{\circ} 34^{\prime}$ de latitude Sul, $40^{\circ} 26^{\prime}$ de longitude Oeste e altitude de $375 \mathrm{~m}$, no período de dezembro de 2000 a novembro de 2001. O clima da região é do tipo semi-árido, segundo a classificação de Köeppen (BRASIL, 1992), caracterizado por apresentar as seguintes médias anuais (médias de 36 anos): temperatura de $26,3^{\circ} \mathrm{C}$, precipitação pluviométrica de 570 $\mathrm{mm}$, umidade relativa do ar de $61,7 \%$ e insolação diá- ria de 7,3 horas, conforme dados da Estação de Meteorologia da Embrapa Semi-Árido.

As plantas foram da cultivar Tommy Atkins com cinco anos de idade, enxertadas em porta-enxerto da variedade Espadinha, distribuídas no espaçamento de $8 \mathrm{~m}$ x 6m (208 plantas/ha). No florescimento e 30 dias após, as plantas foram pulverizadas com pesticida orgânico preparado a partir do fermentado à base de rúmen de bovino. $\mathrm{O}$ sistema de irrigação utilizado foi do tipo microaspersão com um emissor por planta, uma vazão de $90 \mathrm{~L} / \mathrm{h}$, turno de rega de dois dias e tempo de irrigação de duas horas, pela tarde.

$\mathrm{O}$ delineamento experimental utilizado foi o inteiramente casualizado em esquema de parcelas subdivididas no tempo. As parcelas foram constituídas por dez tratamentos em esquema fatorial $4 \times 2+2$, sendo quatro doses de PBZ foliar, duas formas de aplicação e dois tratamentos adicionais, que foram uma aplicação de PBZ no solo e uma aplicação foliar de água. As subparcelas foram representadas pelas épocas de avaliação $(68$ a 110, 131 a 179 e 190 a 216 dias após a aplicação do PBZ para o florescimento da planta; e 162 a 203, 256 a 287 e 327 a 337 dias após a aplicação de PBZ para o número e produção de frutos por planta). Foram usadas quatro repetições e a parcela (unidade) experimental foi representada por uma planta.

Os tratamentos foram os seguintes: T1 - PBZ aplicado ao solo em única aplicação de $2 \mathrm{~mL}$ do i.a./planta (dose usada em função do diâmetro da copa da planta, ou seja, 1,0 mL do ingrediente ativo PBZ por 1,0 m de diâmetro de copa), T2 - única aplicação foliar de água, T3 - PBZ via foliar em única aplicação de 0,5 mL do i.a./planta, T4 - PBZ via foliar em duas aplicações de 0,25 mL do i.a./planta, T5 - PBZ via foliar em única aplicação de $1 \mathrm{~mL}$ do i.a./planta, T6 - PBZ via foliar em duas aplicações de 0,5 mL do i.a./planta, T7 - PBZ via foliar em única aplicação de 1,5 mL do i.a./planta, T8 - PBZ via foliar em duas aplicações de $0,75 \mathrm{~mL}$ do i.a./planta, T9 - PBZ via foliar em única aplicação de $2 \mathrm{~mL}$ do i.a./planta e T10 - PBZ via foliar em duas aplicações de $1 \mathrm{~mL}$ do i.a./planta.

A primeira aplicação foi feita em 07/12/00, quando as plantas apresentavam cerca de $70 \%$ dos ramos com 30 dias de idade. A dose de $2 \mathrm{~mL}$ de PBZ foi dissolvida num volume de 2 litros de água e aplicada no solo ao redor do tronco da planta. Em seguida, no mesmo volume de solução, juntamente com espalhante adesivo na dose de $3 \mathrm{~mL} / 20 \mathrm{~L}$ de água, fizeram-se as 
aplicações foliares do PBZ, usando-se o mesmo pulverizador com um bico tipo cone cheio. No dia 24/01/01 (48 dias após a primeira aplicação de PBZ), fez-se a segunda aplicação foliar do PBZ nos tratamentos que tiveram as doses divididas.

Decorridos 113 dias após a primeira aplicação do PBZ, quando as plantas apresentavam ramos maduros (algumas em início de florescimento), foram iniciadas quatro pulverizações foliares semanais de nitrato de cálcio a 2,5\% em todos os tratamentos.

Em cada tratamento foram determinadas as variáveis percentuais de florescimento, número de frutos e produção por planta. $O$ percentual de florescimento foi obtido mediante observações visuais, atribuindo-se um valor de 0 a $100 \%$ para o florescimento em cada lado da planta (Norte, Sul, Leste e Oeste), e calculando-se a média. Na época da colheita, foram contados os frutos, além da produção em quilogramas por planta. Os dados foram submetidos à análise de variância e as médias dos tratamentos, comparadas pelo teste de Tukey a 5\% de probabilidade, utilizando-se o programa estatístico SISVAR - Sistema de Análise de Variância para Dados Balanceados, desenvolvido por Ferreira (2000).

\section{RESULTADOS E DISCUSSÃO}

Pela análise de variância, evidenciaram-se efeitos significativos para as causas de variação adicional (PBZ no solo $x$ testemunha sem PBZ), época de aplicação, adicional x época e para o contraste fatorial vs adicional (dose $\mathrm{x}$ número de aplicações) com relação às variáveis percentual de florescimento, número de frutos e produção por planta (Tabela 1). Houve também efeito significativo para a dose do PBZ foliar com relação à variável florescimento da planta.

TABELA 1 - Resumo da análise de variância do percentual de florescimento (\%), número de frutos por planta e produção por planta $(\mathrm{kg})$ em mangueira da variedade Tommy Atkins, em função da aplicação de PBZ. Petrolina, PE. 2001.

\begin{tabular}{|c|c|c|c|c|}
\hline \multirow{2}{*}{ Causas de variação } & \multirow{2}{*}{ GL } & \multicolumn{3}{|c|}{ QM } \\
\hline & & Florescimento & $\mathbf{N}^{\mathbf{0}}$ Frutos & Produção \\
\hline Dose & 3 & $380,2326 *$ & $3.454,5972$ & 945,8979 \\
\hline Número de aplicações & 1 & 123,7604 & $2.442,0416$ & 470,3333 \\
\hline Dose $\mathrm{x}$ tipo & 3 & 78,6493 & $2.702,7083$ & 589,8569 \\
\hline Adicional & 1 & $1.107,0416 *$ & $15.100,1666 *$ & $2.414,6222 *$ \\
\hline Fatorial vs adicional & 1 & $1.089,0187 *$ & $17.304,0083 *$ & $2.285,2958 *$ \\
\hline Erro a & 30 & 79,0722 & $1.895,8611$ & 387,5404 \\
\hline Época & 2 & $4.119,0083 *$ & $49.710,0333 *$ & $10.346,4116 *$ \\
\hline Dose $\mathrm{x}$ época & 6 & 75,2118 & 905,8993 & 206,2234 \\
\hline Tipo x época & 2 & 132,2604 & $2.047,5729$ & 578,6268 \\
\hline Dose $\mathrm{x}$ tipo $\mathrm{x}$ época & 6 & 433,4409 & $5.841,7812$ & 967,4387 \\
\hline Adicional x época & 2 & $1.830,1666 *$ & $25.145,0416 *$ & $3.873,1397 *$ \\
\hline Erro b & 60 & 152,2305 & $2.375,1277$ & 430,8745 \\
\hline Total & 119 & & & \\
\hline $\mathrm{CV}(\%)$ & & 71,80 & 84,29 & 76,63 \\
\hline Média & & 17,18 & 57,81 & 27,08 \\
\hline
\end{tabular}

\footnotetext{
*Significativo a $5 \%$ pelo teste $\mathbf{F}$
} 


\section{Percentual de florescimento}

No período de avaliação, ocorreram três surtos de florescimento. A intensidade de florescimento foi maior na segunda época (131 a 179 dias após a primeira aplicação de PBZ), atingindo média geral de $27,68 \%$, enquanto a primeira (68 a 110 dias após) e a terceira (190 a 216 dias após) épocas atingiram respectivamente médias de $8,95 \%$ e $14,42 \%$ (Tabela 2).

A maior intensidade de florescimento dos ramos na segunda época foi atribuída aos tratamentos aplicados e às pulverizações de nitrato de cálcio para quebrar a dormência das gemas, apesar de que a primeira época de florescimento ocorreu antes da aplicações do nitrato. De acordo com Núnez-Elisea e Davenport (1995), a maturidade do ramo ou a idade da gema é importante para que ocorra tempo suficiente para a diferenciação da gema vegetativa em gema floral. Com a aplicação de PBZ (16 g/planta), foi observado $100 \%$ de desenvolvimento de gemas florais da variedade Kheiw Sawoey aos 112 dias após a aplicação (TONGUMPAI et al., 1996).

Os percentuais de florescimento dos tratamentos diferiram significativamente para a segunda e terceira épocas (Tabela 2). Na segunda época, a aplicação do PBZ no solo determinou percentual de florescimento $(63,75 \%)$ superior aos demais tratamentos estudados, que não diferiram estatisticamente entre si. Na terceira época, os tratamentos de 0,25 $\mathrm{mL}$ de PBZ, em duas aplicações foliares, e de $2 \mathrm{~mL}$ de PBZ, em única aplicação foliar, determinaram os valores maiores.

Com relação ao modo de aplicação de PBZ na planta, vários autores consideraram a aplicação no solo mais eficiente que a foliar (VOON et al., 1991; BURONDKAR et al., 1996; AGUIAR, 2001) no florescimento da planta. Quanto às pulverizações foliares de PBZ, foi observado por Tongumpai et al. (1996) que aplicações de 1000 e 2000 mg/L induziram maior número de panículas florais e um florescimento mais uniforme após 102 dias do ínicio do tratamento. Também, em outro estudo, a dose de $2000 \mathrm{mg} / \mathrm{L}$ apresentou maior número de inflorescências acumuladas que a dose de $1000 \mathrm{mg} / \mathrm{L}$ e a testemunha (VOON et al., 1991); entretanto, outros autores não encontraram respostas semelhantes (REIS et al., 2000).

TABELA 2 - Percentuais de florescimento de ramos de mangueiras Tommy Atkins obtidos nos tratamentos com aplicação de PBZ. Petrolina, PE. 2001.

\begin{tabular}{|c|c|c|c|}
\hline \multirow{3}{*}{ Tratamentos } & \multicolumn{3}{|c|}{ Épocas de Florescimento (dias após a $1^{\mathrm{a}}$ aplicação de PBZ) } \\
\hline & $1^{\mathrm{a}}$ & $2^{\mathrm{a}}$ & $3^{\mathrm{a}}$ \\
\hline & 68 a 110 & 131 a 179 & 190 a 216 \\
\hline 1) $2 \mathrm{~mL}$ solo & $25,00 \mathrm{a}$ & $63,75 \mathrm{a}$ & $1,25 \mathrm{~b}$ \\
\hline 2) Água foliar & $11,25 \mathrm{a}$ & $20,00 \mathrm{~b}$ & $18,00 \mathrm{~b}$ \\
\hline 3) $0,5 \mathrm{~mL}$ foliar & $7,50 \mathrm{a}$ & $18,75 \mathrm{~b}$ & $11,25 \mathrm{~b}$ \\
\hline 4) $0,25+0,25 \mathrm{~mL}$ foliar & $5,00 \mathrm{a}$ & $32,5 \mathrm{~b}$ & $30,00 \mathrm{a}$ \\
\hline 5) $1 \mathrm{~mL}$ foliar & $0,00 \mathrm{a}$ & $26,25 \mathrm{~b}$ & $5,00 \mathrm{~b}$ \\
\hline 6) $0,5+0,5 \mathrm{~mL}$ foliar & $4,25 \mathrm{a}$ & $15,00 \mathrm{~b}$ & $15,00 \mathrm{~b}$ \\
\hline 7) $1,5 \mathrm{~mL}$ foliar & $9,00 \mathrm{a}$ & $18,00 \mathrm{~b}$ & $12,50 \mathrm{~b}$ \\
\hline 8) $0,75+0,75 \mathrm{~mL}$ foliar & $7,50 \mathrm{a}$ & $28,75 \mathrm{~b}$ & $7,50 \mathrm{~b}$ \\
\hline 9) $2 \mathrm{~mL}$ foliar & $11,25 \mathrm{a}$ & $16,25 \mathrm{~b}$ & $31,25 \mathrm{a}$ \\
\hline 10) $1+1 \mathrm{~mL}$ foliar & $8,75 \mathrm{a}$ & $37,50 \mathrm{~b}$ & $12,50 \mathrm{~b}$ \\
\hline Media (\%) & $8,95 \mathrm{C}$ & $27,68 \mathrm{~A}$ & $14,42 \mathrm{~B}$ \\
\hline
\end{tabular}

Médias seguidas pela mesma letra minúscula nas colunas e maiúsculas nas linhas não diferem estatisticamente entre si a $5 \%$ de probabilidade pelo teste de Tukey.

Ciênc. agrotec., Lavras, v. 28, n. 4, p. 807-814, jul./ago., 2004 
A primeira época de florescimento do tratamento com PBZ no solo iniciou-se aos 68 dias após o início do experimento e prosseguiu até o $100^{\circ}$ dia (Tabela 3), correspondendo a $25 \%$ do florescimento da planta nesse tratamento (Tabela 2). Para os tratamentos de água e PBZ $(0,25 \mathrm{~mL}$ em duas aplicações) foliares, a primeira época de florescimento iniciou-se aos 82 dias após o início do experimento e prosseguiu até $110^{\circ}$ dia (Tabela 3), correspondendo a $11,25 \%$ e $5 \%$ de florescimento, respectivamente, nesses tratamentos (Tabela 2). A segunda época de florescimento correspondeu a $63,75 \%$ para o PBZ no solo, $20 \%$ para água foliar e $32,5 \%$ para o PBZ foliar.

Essa época iniciou o florescimento aos 131 dias após o início do experimento, prosseguindo até o $161^{\circ}$ dia para o tratamento com PBZ no solo. Para a água e PBZ foliares, foi iniciado aos 154 dias após, prosseguindo até o $179^{\circ}$ dia. A terceira época de florescimento iniciou-se aos 190 dias após o início, indo até o $216^{\circ}$ dia para os três tratamentos e correspondeu a $1,25 \%, 18 \%$ e $30 \%$ de florescimento da planta, respectivamente para os tratamentos PBZ no solo, água e PBZ foliar.

Na primeira época, pôde ser verificado que a aplicação do PBZ no solo antecipou o florescimento por duas semanas em relação às aplicações foliares de água e PBZ. Na segunda época de florescimento, houve uma antecipação maior, de 23 dias. Além disso, verificou-se que ocorreu uma maior concentração do florescimento com a aplicação do PBZ no solo nas duas primeiras épocas em relação às aplicações foliares de água e PBZ, que concentraram mais nas duas últimas épocas.

De acordo com Burondkar et al. (1996), o método de aplicação do produto no solo em volta do tronco é fácil e mais efetivo, entre todos os métodos, inclusive o foliar. Além disso, o PBZ aplicado no solo permitiu antecipar o florescimento da planta e, em alguns casos, quanto maior a dose usada no solo, maior foi a antecipação do florescimento, chegando a 16 dias com 2,5 g/planta e 40 dias com $40 \mathrm{~g} / \mathrm{planta}$ da variedade Tommy Atkins em relação à testemunha (SALAZARGARCIA e VASQUEZ-VALDIVIA, 1997). Além da precocidade, a aplicação de PBZ no solo proporcionou aumento de $75,75 \%$ de florescimento dos ramos da mangueira da variedade Nam Dok Mai (CHARNVICHIT et al., 1996, citado por AGUIAR, 2001).

\section{Número de frutos por planta}

A segunda época de colheita (256 a 287 dias após a $1^{\mathrm{a}}$ aplicação de $\mathrm{PBZ}$ ) produziu número significativamente maior de frutos (96 frutos/planta) em comparação com a primeira (27 frutos/planta) e a terceira (51 frutos/planta) épocas (Tabela 4).

A maior quantidade de frutos colhidos na segunda época foi atribuída ao efeito dos tratamentos aplicados e das pulverizações de nitrato de cálcio para quebrar a dormência das gemas, possibilitando maior percentual de florescimento e, conseqüentemente, maior número de frutos. O PBZ aplicado no solo (2 $\mathrm{mL}$ do i.a./planta) foi superior aos demais tratamentos na segunda época de colheita, obtendo-se uma diferença de 119 frutos/planta em relação ao segundo melhor tratamento (Tabela 4). Os tratamentos com aplicação foliar de PBZ não superaram estatisticamente a testemunha em nenhuma época de colheita.

TABELA 3 - Início e fim das épocas de florescimento da mangueira 'Tommy Atkins' submetidas aos tratamentos do $2 \mathrm{~mL}$ do i.a. de PBZ no solo, água foliar e $0,5 \mathrm{~mL}$ do i.a. (duas aplicações de 0,25mL) de PBZ foliar. Petrolina, PE, 2001.

\begin{tabular}{|c|c|c|c|}
\hline \multirow{3}{*}{ Tratamento } & \multicolumn{3}{|c|}{ Épocas de Florescimento (dias após início do experimento) } \\
\hline & $\mathbf{1}^{\mathrm{a}}$ & $2^{\mathrm{a}}$ & $3^{\mathbf{a}}$ \\
\hline & Início - Fim & Início - Fim & Início - Fim \\
\hline $2 \mathrm{~mL}$ de PBZ no solo & $68-100$ & $131-161$ & $190-216$ \\
\hline Água foliar & $82-110$ & $154-179$ & $190-216$ \\
\hline $0,25+0,25 \mathrm{~mL}$ de PBZ foliar & $82-110$ & $154-179$ & $190-216$ \\
\hline
\end{tabular}


Esses resultados confirmam os alcançados em outros estudos (IYER e KURIAN, 1992; BURONDKAR et al., 1996), exceto no de Oosthuyse e Jacobs (1996), que não constataram aumento no número de frutos colhidos em mangueiras tratadas com PBZ aplicado no solo.

\section{Produção por planta}

Os resultados obtidos para essa variável (Tabela 5) foram similares aos resultados para a variável número de frutos por planta.
A segunda época de produção (256 a 287 dias após a $1^{\text {a }}$ aplicação de PBZ), com uma produção média de $44,74 \mathrm{~kg} /$ planta, foi significativamente superior à primeira $(13,25 \mathrm{~kg} /$ planta $)$ e à terceira $(23,27 \mathrm{~kg} /$ planta) épocas. O PBZ aplicado no solo superou os demais tratamentos na segunda época, obtendo uma diferença de $40,75 \mathrm{~kg}$ de frutos/planta em relação ao segundo melhor tratamento. Esses resultados confirmam os alcançados em outros estudos com PBZ aplicado no solo (IYER e KURIAN, 1992; BURONDKAR et al., 1996).

TABELA 4 - Número de frutos colhidos por mangueira ‘Tommy Atkins' em função de diversos tratamentos com aplicação de PBZ. Petrolina, PE, 2001.

\begin{tabular}{|c|c|c|c|}
\hline \multirow{3}{*}{ Tratamento } & \multicolumn{3}{|c|}{ Épocas de Colheita (dias após a $1^{\text {a }}$ aplicação de PBZ) } \\
\hline & $\mathbf{1}^{\mathrm{a}}$ & $2^{\mathrm{a}}$ & $3^{\mathrm{a}}$ \\
\hline & 162 a 203 & 256 a 287 & 327 a 337 \\
\hline 1) $2 \mathrm{~mL}$ solo & $76 \mathrm{a}$ & $242 \mathrm{a}$ & $3 \mathrm{a}$ \\
\hline 2) Água foliar & $32 \mathrm{a}$ & $77 \mathrm{~b}$ & $61 \mathrm{a}$ \\
\hline 3) $0,5 \mathrm{~mL}$ foliar & $21 \mathrm{a}$ & $63 \mathrm{~b}$ & $42 \mathrm{a}$ \\
\hline 4) $0,25+0,25 \mathrm{~mL}$ foliar & 13 a & $120 \mathrm{~b}$ & $115 \mathrm{a}$ \\
\hline 5) $1 \mathrm{~mL}$ foliar & $0 \mathrm{a}$ & $101 \mathrm{~b}$ & $20 \mathrm{a}$ \\
\hline 6) $0,5+0,5 \mathrm{~mL}$ foliar & $10 \mathrm{a}$ & $35 \mathrm{~b}$ & $54 \mathrm{a}$ \\
\hline 7) $1,5 \mathrm{~mL}$ foliar & $32 \mathrm{a}$ & $56 \mathrm{~b}$ & $56 \mathrm{a}$ \\
\hline 8) $0,75+0,75 \mathrm{~mL}$ foliar & $24 \mathrm{a}$ & $97 \mathrm{~b}$ & $23 \mathrm{a}$ \\
\hline 9) $2 \mathrm{~mL}$ foliar & $36 \mathrm{a}$ & $46 \mathrm{~b}$ & $88 \mathrm{a}$ \\
\hline 10) $1+1 \mathrm{~mL}$ foliar & $23 \mathrm{a}$ & $123 \mathrm{~b}$ & $46 \mathrm{a}$ \\
\hline Média & $27 \mathrm{C}$ & $96 \mathrm{~A}$ & $51 \mathrm{~B}$ \\
\hline
\end{tabular}

Médias seguidas pela mesma letra minúscula nas colunas e maiúsculas nas linhas não diferem estatisticamente entre si a $5 \%$ de probabilidade pelo teste de Tukey

Ciênc. agrotec., Lavras, v. 28, n. 4, p. 807-814, jul./ago., 2004 
TABELA 5 - Produção da mangueira ‘Tommy Atkins’ (kg/planta) em resposta a vários tratamentos com PBZ. Petrolina, PE, 2001.

\begin{tabular}{|c|c|c|c|}
\hline \multirow{3}{*}{ Tratamento } & \multicolumn{3}{|c|}{ Épocas de Produção } \\
\hline & $\mathbf{1}^{\mathrm{a}}$ & $2^{\mathrm{a}}$ & $3^{\mathrm{a}}$ \\
\hline & 162 a 203 & 256 a 287 & 327 a 337 \\
\hline 1) $2 \mathrm{~mL}$ solo & 36,79 a & 99,63 a & $5,13 \mathrm{a}$ \\
\hline 2) água foliar & 15,86 a & $36,00 \mathrm{~b}$ & 25,50 a \\
\hline 3) $0,5 \mathrm{~mL}$ foliar & $9,63 \mathrm{a}$ & $30,88 \mathrm{~b}$ & 20,33 a \\
\hline 4) $0,25+0,25 \mathrm{~mL}$ foliar & $7,95 \mathrm{a}$ & $58,88 \mathrm{~b}$ & $51,95 \mathrm{a}$ \\
\hline 5) $1 \mathrm{~mL}$ foliar & $0,00 \mathrm{a}$ & $42,63 \mathrm{~b}$ & $8,25 \mathrm{a}$ \\
\hline 6) $0,5+0,5 \mathrm{~mL}$ foliar & $4,24 \mathrm{a}$ & $20,50 \mathrm{~b}$ & 22,16 a \\
\hline 7) $1,5 \mathrm{~mL}$ foliar & $17,41 \mathrm{a}$ & $29,25 \mathrm{~b}$ & $26,25 \mathrm{a}$ \\
\hline 8) $0,75+0,75 \mathrm{~mL}$ foliar & $12,02 \mathrm{a}$ & $48,63 \mathrm{~b}$ & 11,73 a \\
\hline 9) $2 \mathrm{~mL}$ foliar & $18,04 \mathrm{a}$ & $25,38 \mathrm{~b}$ & 44,18 a \\
\hline 10) $1+1 \mathrm{~mL}$ foliar & $10,53 \mathrm{a}$ & $55,63 \mathrm{~b}$ & $21,25 \mathrm{a}$ \\
\hline Media & $13,25 \mathrm{C}$ & $44,74 \mathrm{~A}$ & $23,27 \mathrm{~B}$ \\
\hline
\end{tabular}

Médias seguidas pela mesma letra minúscula nas colunas e maiúsculas nas linhas não diferem estatisticamente entre si a $5 \%$ de probabilidade pelo teste Tukey.

\section{CONCLUSÕES}

O PBZ aplicado ao solo determinou percentuais de florescimento, número de frutos e produção por planta superiores à testemunha (água foliar) e ao PBZ foliar, além de antecipar o florescimento de mangueiras 'Tommy Atkins'.

O PBZ foliar, independente do número de aplicações e das doses aplicadas, não superou a testemunha em relação ao percentual de florescimento, número de frutos e produção por planta.

O PBZ aplicado no solo antecipou o florescimento em 23 dias, em relação à testemunha e ao PBZ foliar, na principal época de florescimento.

\section{REFERÊNCIAS BIBLIOGRÁFICAS}

AGUIAR, P.A. A. Noções teóricas e práticas sobre o florescimento da manga. Petrolina, PE: [s.n.], 2001. 102 p.

BRASIL. Departamento Nacional de Meteorologia. Serviço de Produção de Informação. Normas climatológicas: 1961 - 1990. Brasília: SPI/EMBRAPA, 1992. 84 p.
BURONDKAR, M. M.; GUNJATE, R. T.; MAGDUM, M. B.; GOVEKAR, M. A.; WAGHMARE, G. M. Increasing productivity of mango orchards by pruning and application of paclobutrazol. Acta Horticulturae, Amsterdam, p. 367-374, 1996.

FERREIRA, D. F. Análises estatísticas por meio do Sisvar para Windows: versão 4.0. In: REUNIÃO ANUAL DA REGIÃO BRASILEIRA DA SOCIEDADE INTERNACIONAL DE BIOMETRIA, 45., 2000, São Carlos, SP. Anais... São Carlos: UFSCar, 2000. p. 255-258.

IYER, C. P. A.; KURIAN, R. M. Tree size control in mango (Mangifera indica L.): some considerations. Acta Horticulturae, Amsterdam, n. 321, p. 425-436, 1992.

NÚNEZ-ELISEA, R.; DAVENPORT, T. L. Effect of leaf age, duration of cool temperature treatment, and photoperiod on bud dormancy release and floral initiation in mango. Scientia Horticulturae, Amsterdam, v. 62 , n. $1 / 2$, p. $62-63,1995$. 
OOSTHUYSE, S. A.; JACOBS, G. Effect of soil applied paclobutrazol on the retention, fruit size, tree yield and tree revenue in 'Sensation' and 'Tommy Atkins' mango. Acta Horticulturae, Amsterdam, n. 296, p. 431-440, 1996.

REIS, V. C. S.; CASTRO NETO, M. T. de; SOARES J. M. Efeito da aplicação foliar do paclobutrazol na floração e frutificação da mangueira (Mangifera indica L.) cv. 'Tommy Atkins'. Magistra, Cruz das Almas, v. 12, n. 1/2, p. 11-18, jan./jun. 2000.

SALAZAR-GARCIA, S.; VAZQUEZ-VALDIVIA, V. Physiological persistence of paclobutrazol on the
'Tommy Atkins' mango (Mangifera indica L. ) under rainfed conditions. Journal of Horticultural Science, Ashford, v. 72, n. 2, p. 339-345, Mar. 1997.

TONGUMPAI, P.; CHANTAKULCHAN, K.; SUBHADRABANDHU, S.; OGATA, R. Foliar aplication of paclobutrazol on flowering of mango. Acta Horticulturae, Amsterdam, n. 296, p. 175179, 1996.

VOON, C. H.; PITAKPAIVAN, C.; TAN, S. J. Mango cropping manipulation with cultar. Acta Horticulturae, Amsterdam, n. 291, p. 219-228, 1991. 